\title{
Contribution à la cartographie des potentialités mellifères du Haut-Jura; exemple de variations spatio-temporelles autour d'un rucher à Chaumont/NE
}

\section{Résumé}

200 espèces végétales apicoles sont recensées et suivies phénologiquement durant 4 ans dans une contrée du HautJura. Sur la base de ces variations spatio-temporelles, l'auteur distingue 5 saisons mellifères principales et leurs différentes ressources. Quelques propositions sont suggérées pour aménager et exploiter rationnellement le paysage végétal.

\begin{abstract}
During 4 years, 200 melliferous species have been inventoried and examined phenologically in an area of the Swiss High Jura. On the basis of their spatio-temporal variations, the author distinguishes five melliferous seasons with their corresponding resources, and makes a number of proposals how to manage the melliferous landscape.
\end{abstract}

\section{Zusammenfassung}

Während vier Jahren wurden in einer Gegend des Haut-Jura 200 honigtragende Arten gezählt und phänologisch untersucht. Aufgrund raumzeitlicher Veränderungen unterscheidet der Verfasser fünf Hauptperioden mit ihren entsprechenden Ressourcen und macht einige Vorschläge, wie die Landschaft vernünftig zu planen und zu bewirtschaften ist.

\section{Introduction}

Si la biologie de l'abeille est relativement bien connue (notamment son action pollinisatrice), son écologie en relation avec l'environnement végétal reste méconnue. Dans leurs récents et remarquables essais de cartographie et d'évaluation des potenialités mellifères en Roussillon et en Haute-Garonne, BRIANE et GABROL (1986) ainsi que BALAYER (1990) ont compris l'intérêt socioéconomique d'une meilleure exploitation des ressources apicoles: problèmes de transhumance et définition des "crus» de miel. En fonction de la phénologie des espèces mellifères dans un espace donné, les auteurs cités établissent des cartes de miellées, des indices mellifères et des carences saisonnières.

Dans un but identique mais comprenant également des questions d'aménagement et de gestion du paysage végétal, nous proposons une étude complémentaire à grande échelle (1:5000) dans une zone biogéographique différente.

\section{Cadre d'étude}

Le secteur étudié se situe dans le Jura central, sur le premier anticlinal de Chaumont au-dessus de Neuchâtel (Suisse). La colonie de ruches repose près du sommet de la voûte calcaire (kimméridgien) à l'altitude de $1175 \mathrm{~m}$. La zone cartographiée $\left(1 / 2 \mathrm{~km}^{2}\right)$ correspond à un rayon d'action d'environ $500 \mathrm{~m}$. Au-delà, nous estimons que le butinage n'est plus efficace. Cette remarque est également valable pour les aires de butinage situées à plus de $50 \mathrm{~m}$ de dénivellation au-dessous des ruches.

A l'origine, tout le domaine étudié était de la hêtraie à sapin (Abieti-Fagetum). L'homme, en exploitant cette couverture sylvestre, en cultivant extensivement puis plus intensivement les terres agricoles, en développant finalement le tourisme et les zones de construction, a considérablement modifié le paysage végétal de la région (GEHU 1979, BÉGUIN 1990). Il a augmenté le degré d'artificialisation ou d'hémérobie (littéralement d'apprivoisement) de cette végétation climacique.

La photographie aérienne (fig. 1) et la carte topographique traduisent le mésorelief et les principales structures végétales aux environs de la colonie.

Dans cet essai d'analyse détaillée de l'aire de butinage d'un rucher, nous nous sommes efforcés de travailler le plus inductivement possible en terminant par une synthèse cartographique de l'évolution et de la répartition des principales espèces mellifères dans le temps et dans l'espace.

\section{Résultats}

Des 200 espèces mellifères recensées, la moitié sont des espèces plantées (BÉGUIN 1993): concernant la nomenclature scientifique des plantes vasculaires, nous avons SUivi AESCHIMANN \& BURDET (1989).

60 espèces environ présentent une valeur mellifère significative selon nos observations et nos estimations.

Finalement, pour la cartographie spatio-temporelle des saisons mellifères, seule une vingtaine d'espèces offrant un intérêt apicole supérieur (grande production de miellat, de miellée, de nectar, de pollen et de propolis) ont été retenues.

Claude Béguin, Institut de Géographie, Université de Fribourg, Pérolles, 1700 Fribourg. 


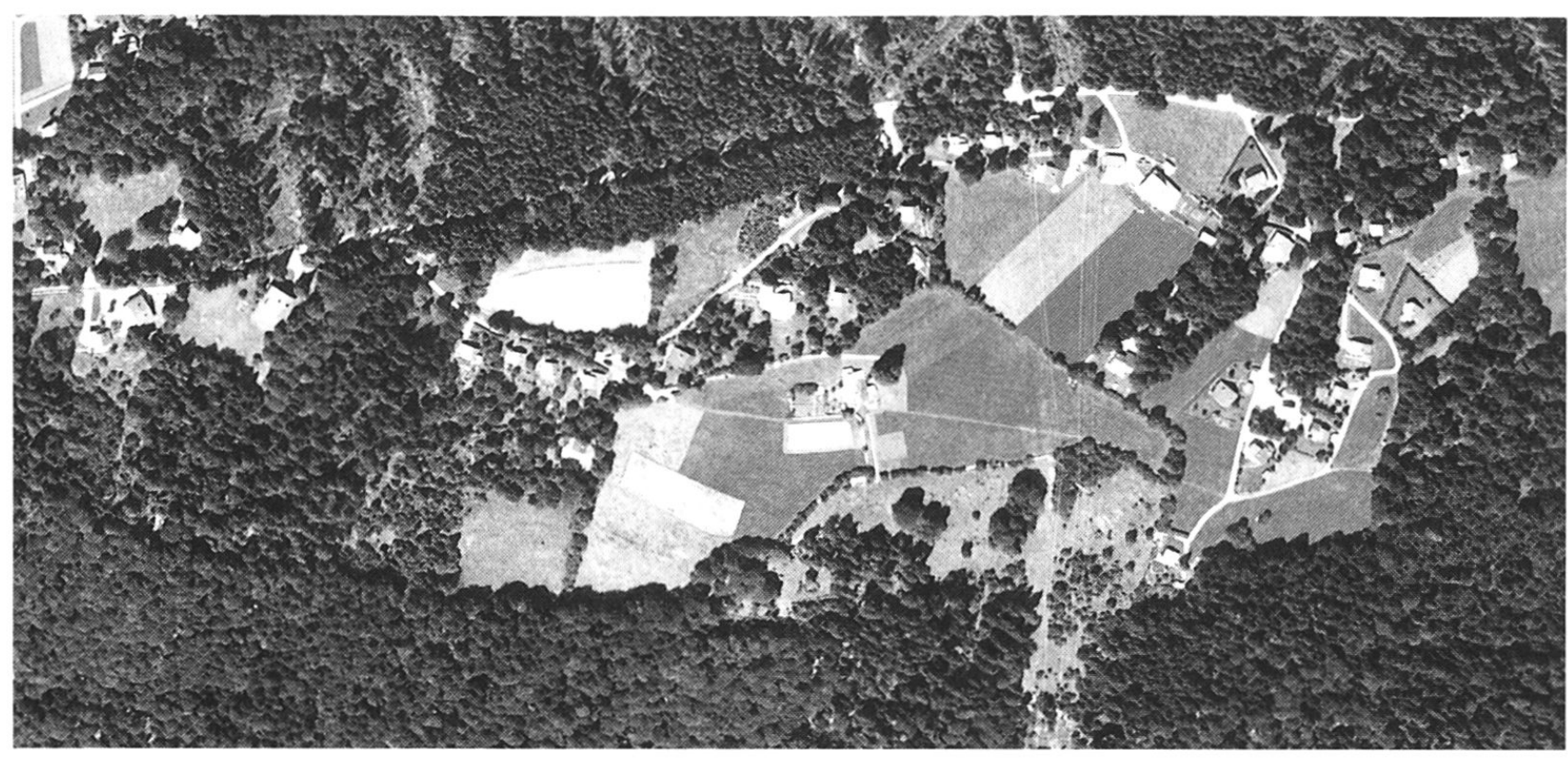

Fig. 1 Physionomie, structures et état du paysage végétal en 1987 dans la région étudiée: forêts, cultures, pâtures, voies de communication et constructions diverses forment une mosaïque comprenant des haies, des lisières, des jachères, des jardins, des bosquets, des pâturages boisés et des parcs arborisés.

Dès le Moyen Age, c'est l'ouverture de la "grande forêt" qui permet la production de miel dans le Haut-Jura. Ce miel très apprécié pourrait encore être exploité plus rationnellement si l'on tenait mieux compte des potentialités mellifères actuelles (aménagements forestiers et aménagements agricoles judicieux).

Photographie de l'Office fédéral de topographie.

Les cartes de saisons mellifères (fig. 2 à 7) sont élaborées sur la base d'observations phénologiques sur le terrain durant les années 1990, 1991, 1992 et 1993. Elles intègrent la floraison des principales espèces mellifëres dans le temps et dans l'espace.

$90 \%$ environ de la surface cartographiée apparait mellifëre: $45 \%$ pour les forêts, $40 \%$ pour les prairies et les pâturages, $5 \%$ pour les jardins s. 1 . Les $10 \%$ non mellifëres se répartissent pour moitié en une zone non végétalisée (constructions diverses, voies de communication) et en une zone végétalisée de cultures représentant un véritable désert biologique (champs d'orge et d'avoine essentiellement).

Les cartes ci-dessous précisent à quels endroits et à quels moments de la période de végétation les abeilles butineuses sont particulièrement actives. Elles donnent une idée assez exacte des profils végétaux de la zone de production.

\section{I) Février/mars: stimulation de la ponte}

Cette première saison mellifëre est importante. Elle correspond au démarrage du couvain dans la ruche. Les butineuses recherchent les moindres ressources de pollen, de nectar et de propolis. L'espèce végétale primordiale est le noisetier (Corylus avellana) abondant dans les haies en bordure des murs et des murgiers, dans les pâturages boisés, le long des routes et des lisières (fig. 2). Ces secteurs filiformes abritent également l'élébore fétide (Helle- borus foetidus) qui, lorsque les conditions climatiques le permettent, apporte le premier nectar.

Ce territoire ne présente pas de grandes variations altitudinales. Par contre, il offre une opposition de versants $\mathrm{N}-\mathrm{NO} / \mathrm{S}-\mathrm{SE}$ avec des enneigements variés. Les changements d'exposition et de pente augmentent la potentialité mellifëre de la plupart des espèces dans la mesure où ils rallongent leurs phases phénologiques. C'est ainsi que l'abondante production de pollen des noisetiers situés au nord prolonge cette première saison mellifëre en attendant la floraison des crocus (Crocus vernus) et des chatons de saules marsault (Salix caprea) sur le versant sud en contre-bas (fig. 2 et 3 ).

Par ailleurs, la vitalité et la floraison des noisetiers sont plus fortes aux endroits de pleine lumière: c'est le cas des haies entretenues et coupées périodiquement.

En dehors de la corylaie, tout en début d'année, les abeilles vont chercher un appoint non négligeable auprès de quelques espèces plantées aux alentours des habitations (aubrietia, éranthe, perce-neige, nivéole, giroflée, bois gentil; Aubrietia deltoidea, Eranthis hiemalis, Galanthus nivalis, Leucojum vernum, Cheiranthus cheiri, Daphne mezereum). Ces especes peuvent fleurir très tôt aux endroits abrités. Elles sont d'autant plus précieuses qu'elles se situent à proximité du rucher.

Il faut attendre apparemment la deuxième saison mellifëre pour disposer d'importantes ressources de propolis. notamment sur les trembles (Populus tremula). 


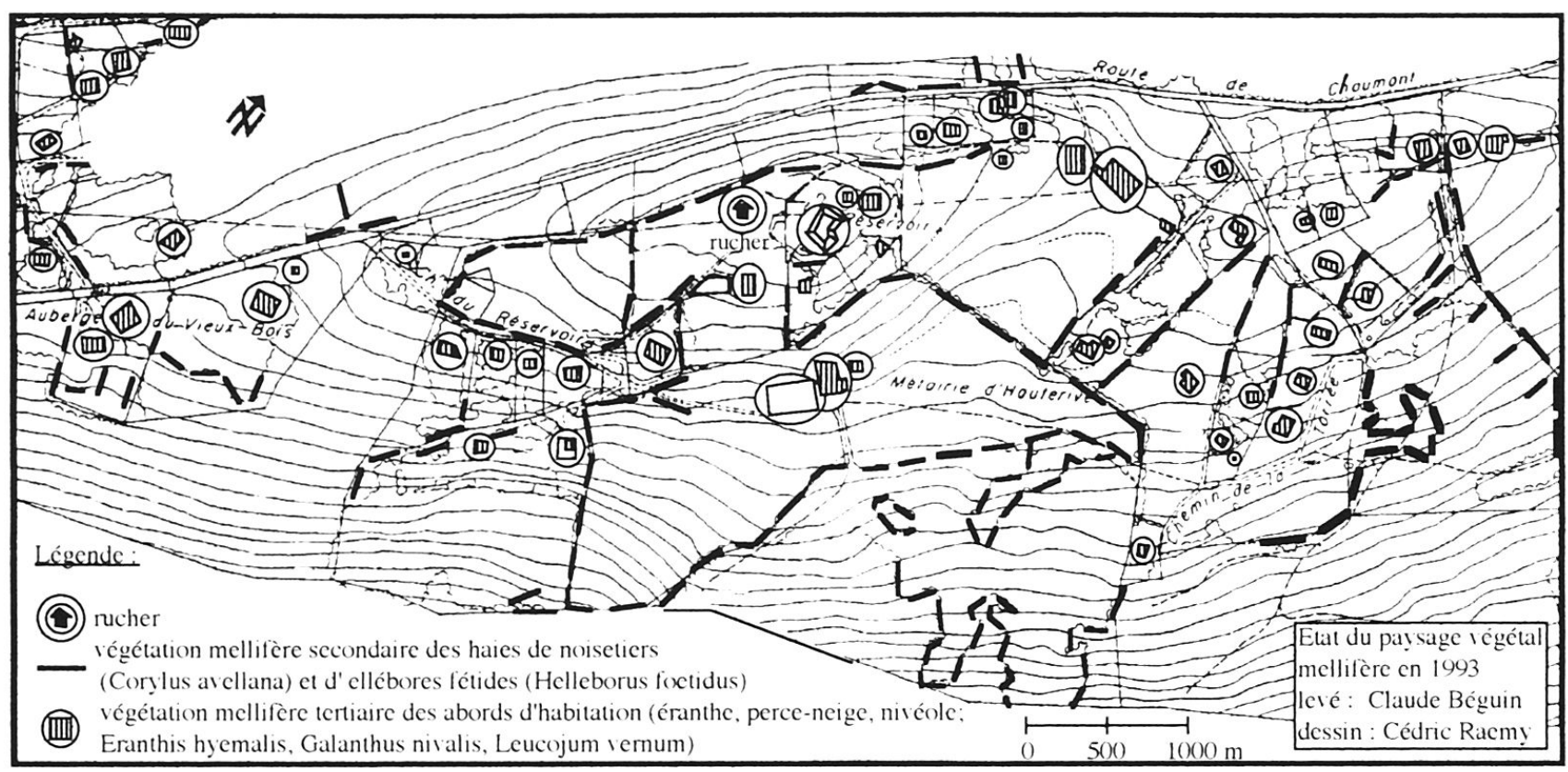

Fig. 2 Ala fin de l'hiver et au premier printemps, la distribution spatiale des espèces mellifères est d'une part linéaire (haies et lisières de noisetiers), d'autre part ponctuelle (60 habitations comprenant des bordures florales, des jardins et des rocailles où fleurissent déjà quelques espèces exotiques très précoces: la bruyère carnée, la bergenie à feuilles charnues, diverses variétés de crocus et/ou de saules, etc.; Erica herbacea, Bergenia crassifolia, Crocus var, Salix sp.)

Reproduit avec l'autorisation du Service cantonal des mensurations cadastrales du 3.12.93.

\section{II) Avril/mi-mai: l'élevage du couvain}

Les conditions climatiques devenant plus clémentes, les butineuses s'activent sur un arbre très attractif pour son pollen et son nectar: le saule marsault ou saule des chèvres (Salix caprea). Les individus mâles et femelles n'ont pas été cartographiés séparément. Par contre, nous avons jugé utile de distinguer les jeunes individus (portant quelques dizaines de chatons) des arbres plus âgés pouvant atteindre une dizaine de mètres de hauteur (portant des centaines de chatons). Cette importante salicacée ne supporte pas la concurrence. Elle croît de préférence en lisière de forêt ou sur des terrains transformés, plus ou moins en friche et plus ou moins ensoleillés. Dans des endroits ponctuels (fig. 3), sa floraison alimentera avant tout les colonies durant le mois d'avril. Son absence dans certaines régions représente vraisemblablement une carence en produits melliferes. Corollairement, sa présence à proximité des ruches est d'autant plus précieuse que les conditions climatiques sont souvent très rudes au premier printemps. Il est vrai cependant que des espèces indigènes moins abondantes, moins attractives et plus dispersées telles que le crocus printanier (Crocus vernus), l'anémone des bois (Anemone nemorosa) et le tabouret des Alpes (Thlaspi alpestre) viennent en quelque sorte renforcer le saule et assurer le relais vers une deuxième source importante de nourriture, à savoir le dent-de-lion (Taraxacum officinale). Il est vrai aussi que certaines es- pèces plantées peuvent jouer un rôle non négligeable avant la floraison de la dent-de-lion notamment dans les zones sans saules. Il s'agit d'espèces telles que le raisin d'ours (Arctostaphyllos uva-ursi), la bruyère (Erica carnea), la petite pervenche (Vinca minor), le groseiller (Ribes uva-crispa), ou les cassissiers (Ribes nigrum, R. rubrum).

\section{III) Mi-mai/mi-juillet: récolte du miel de fleur}

Pourquoi distinguer une période mellifère $\mathrm{mi}-\mathrm{mai} / \mathrm{mi}$ juillet dans le Haut-Jura?

Mi-mai tout d'abord. Début de la production mellifëre maximale: alors que la floraison de la dent-de-lion et celle des premiers arbres fruitiers (cerisiers, merisiers, groseilliers, raisinets, cassis; Prunus avium, Cerasus avium, Ribes uva-crispa, Ribes rubrum, Ribes nigrum) battent encore leur plein, commence à fleurir l'érable sycomore (Acer pseudoplatanus), très attractif et largement répandu, contrairement à l'érable champêtre (Acer campestre) et à l'érable faux-platane (Acer platanoides) relativement rares au-dessus de $1000 \mathrm{~m}$. L'attrait des érables est si fort que les abeilles semblent délaisser des arbustes tels que la viorne lantane (Viburnum lantana), les nerpruns (Rhamnus catharticus, $R$. alpinus) et même les aubépines ( $\mathrm{Cra}$ taegus oxyacantha, C. monogyna). Simultanément, on as- 


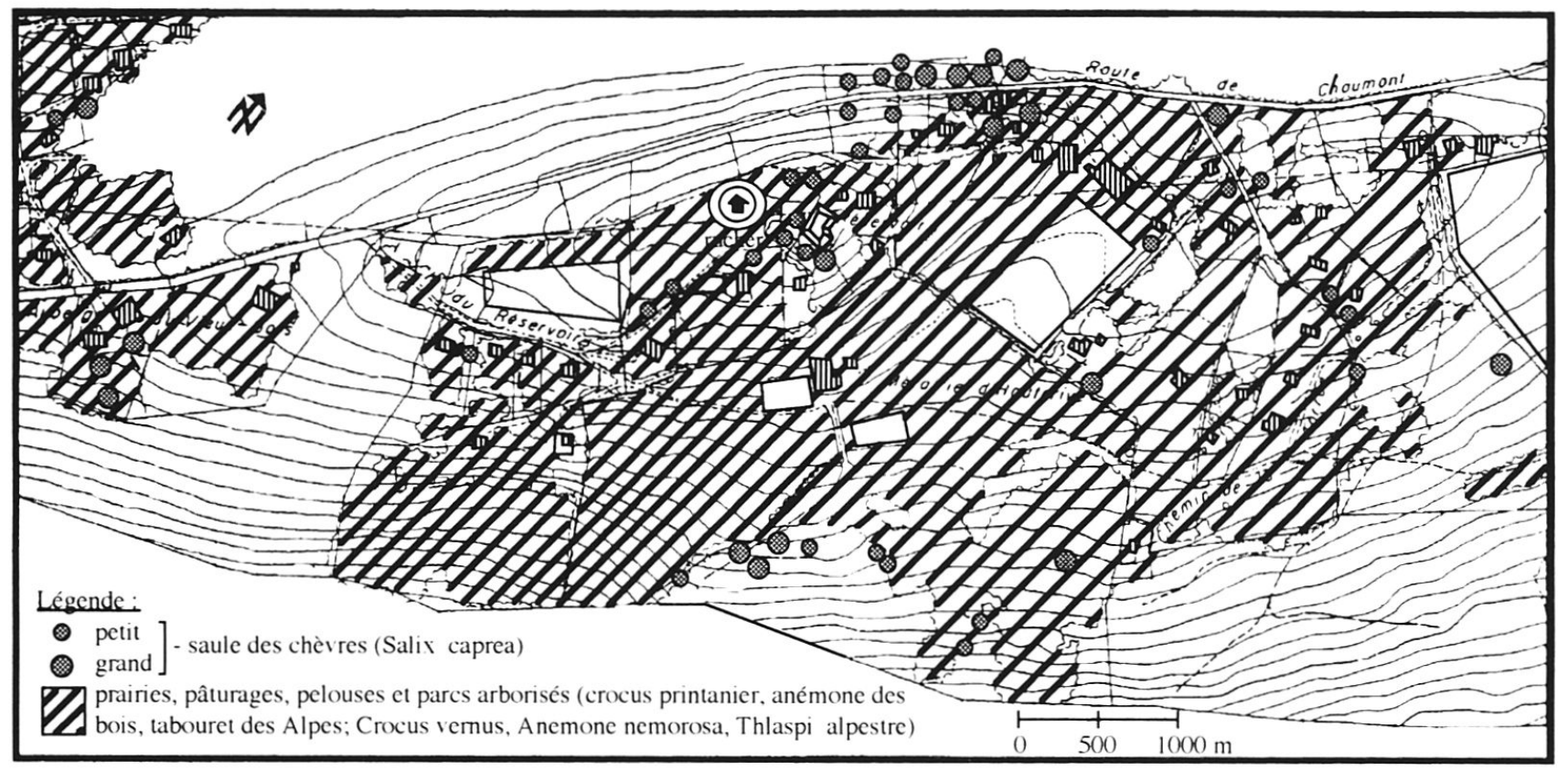

Fig. 3 Les saules arbustifs ne portant que quelques dizaines de chatons sont également cartographiés compte tenu de leur précieux apporten pollen et en nectar au mois d'avril. Le saule des chèvres (Salix caprea) est ici lié aux activités humaines relativement récentes: par exemple, le terrain rajeuni à la suite du remblayage d'une canalisation de gaz (GANSA) sur le versan nord de Chaumont. Ce nouveau sol de nature caillouteuse a été recolonisé dans les années 1980 par une remarquable population de saules des chèvres; sur la carte (au milieu, en haut), on aperçoit l'endroit à forte densité correspondant à la partie supérieure de la tranchée. D'un point de vue apicole, cette cicatrice du paysage se révèle bénéfique.

III. Troisième saison mellifère de MI-MALà MI-JULLET: $1^{\circ}$ récolte de miel de fleur

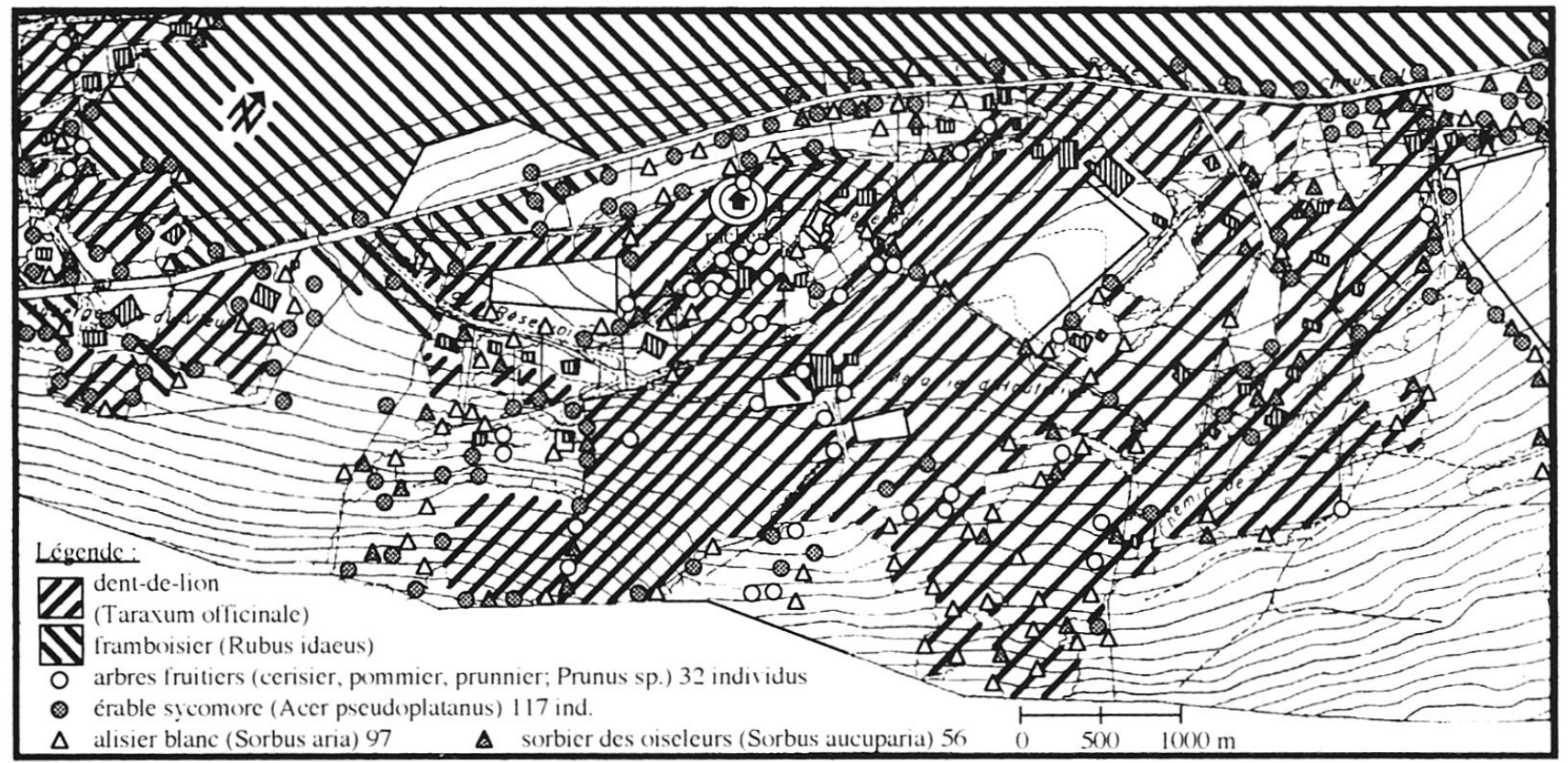

Fig. 4 La figure traduit l'abondance des produits mellifères à la fin du printemps et au début de l'été. On remarque la localisation des framboisiers des bois sur le versant nord: conséquence indirecte des fréquentes tempêtes du nord-ouest qui se sont abattues dans la région ces dernières années. Seule une petite surface d'une ancienne plantation d'épicéas n'a pas encore subi de dégâts par le vent et se développe sans framboisiers.

Tous les arbres cartographiés sont dispersés mais produisent chacun des milliers de fleurs. 
siste à une véritable explosion de la flore mellifère des prairies et des pâturages (hippocrépide, anthyllide, lotier, centaurée, géranium, minette, puis thym, trèfle, chicorée, scabieuse, rhinanthe; Hippocrepis comosa, Anthyllis vulneraria, Lotus corniculatus, Centaurea montana, Geranium silvaticum, Medicago lupulina, Thymus serpyllum, Trifolium montanum, Crepis biennis, Scabiosa columbaria, Rhinanthus alectorolopus).

A ce début d'abondance correspond également une nette amélioration des conditions climatiques (fin des «Saints de Glaces»).

Dans les meilleures conditions les premières hausses ou sections sont posées.

Mi-juillet ensuite. Il est rare que le miellat du puceron Buchneria pectinatae sur le sapin blanc soit disponible avant cette période. D'autre part, à cette date, les foins sont généralement terminés, les regains encore inexistants et les pâturages tondus. Bref, la grande floraison est passée.

C'est par souci de simplification que cette longue période mellifère mi-mai/mi-juillet n'est pas séparée en deux. Mais il est vrai que, dès la mi-juin, après la floraison des derniers arbres fruitiers (pommiers, pruniers; Malus var. Prunussp.), de l'érable (Acer pseudoplatanus), des sorbiers (Sorbus aucuparia, S. aria) et des framboisiers (Rubus idaeus), la nourriture n'est plus très abondante: restent notamment à disposition les trèfles (Trifolium sp.), la gen- tiane jaune (Gentiana lutea), le sureau noir (Sambucus nigra) et les plantains (Plantago media et P. major); s'y ajoute éventuellement le miellat de sapin rouge (Picea excelsa) sans compter évidemment celui tout aussi aléatoire du sapin blanc (Abies alba). La deuxième partie de moindre apport est d'autant mieux individualisée qu'elle correspond non seulement au début de la période de précipitations maximales mais aussi à celle où les abeilles sont très nombreuses à nourrir dans la ruche, soit affaiblies après essaimage.

\section{IV) Mi-juillet/mi-août: récolte du miel de tilleul et de trèfle}

Grâce aux floraisons tardives et décalées des tilleuls, il est encore possible de faire du miel de fleur à cette période. Il s'agit notamment du tilleul à grandes feuilles (Tilia platyphyllos) mais aussi du tilleul à feuilles en cœur (Tilia cordata), de l'hybride (Tilia cordata $\times$ platyphyllos) et plus rarement d'autres tilleuls exotiques: le tilleul d'Amérique (Tilia americana), le tilleul argenté (Tilia tomentosa)', etc. La période de floraison est non seulement prolongée par la diversité des tilleuls (selon PRIMAULT [1971] le tilleul à feuilles en cœur fleurit environ 15 jours plus tard que le tilleul à larges feuilles) mais aussi par l'âge des individus. DEFILA (1992), par exemple, montre qu'à Liestal un tilleul

IV. Quatrième saison mellifère de MI-JUULLET à ML-AOUT: $2^{\circ}$ récolte aléatoire de miel de fleur

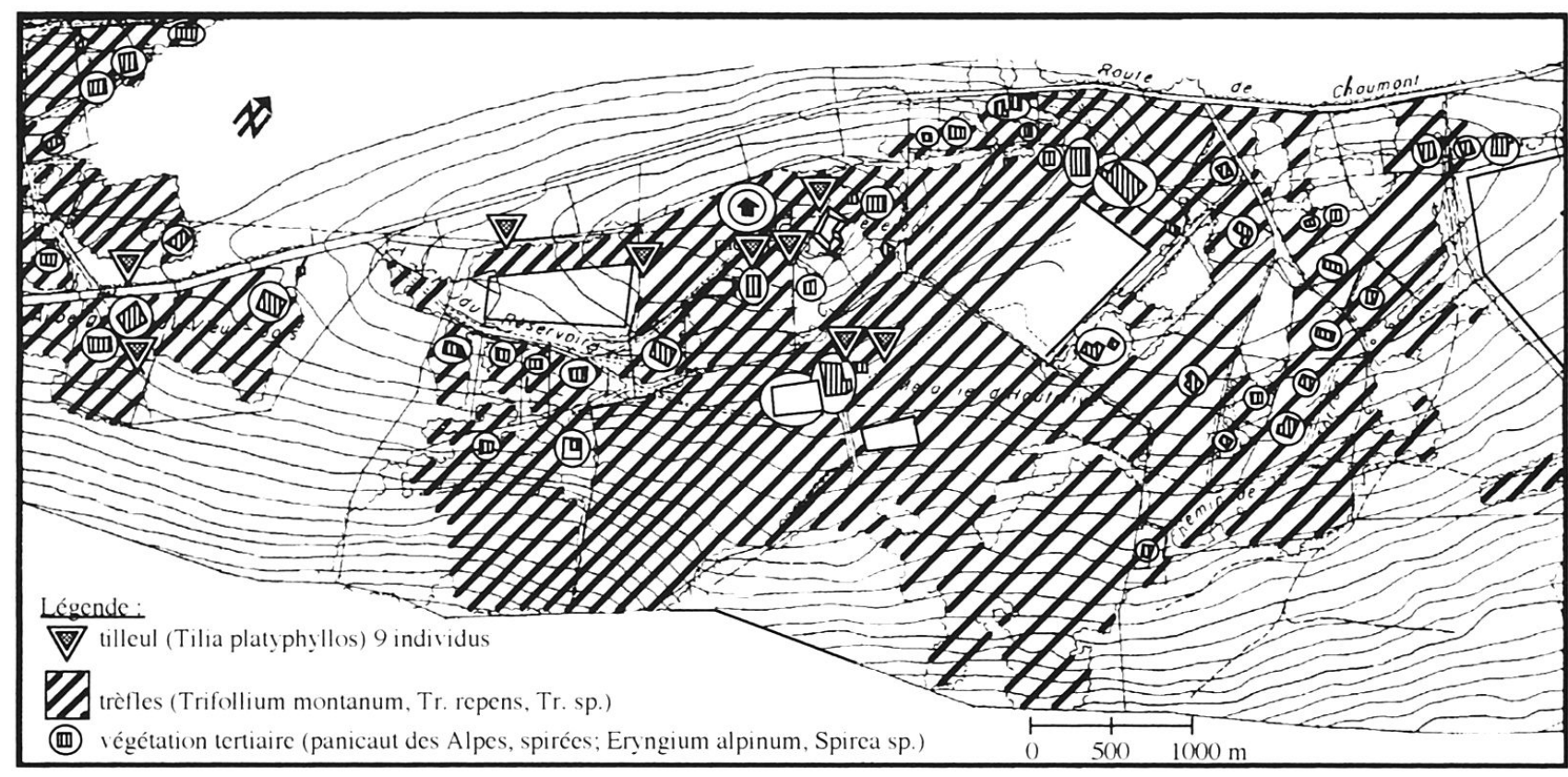

Fig. 5 Les tilleuls à grandes feuilles ont été largement plantés sur la montagne de Chaumont où les conditions climatiques et édaphiques leur dictent chaque année une floraison généralement bonne et accompagnée de miellée. En dehors de la carte mais tout de même à faibles distances, il faut signaler deux allées de tilleuls bordant la route de Chaumont à environ $1 \mathrm{~km}$ au nord-est et $2 \mathrm{~km}$ au sud-ouest du rucher.

Dans des proportions encore non déterminées, le nectar des trèfles dans les zones herbeuses et celui de quelques espèces mellifères des abords d'habitations viennent renforcer cette deuxième récolte plus ou moins aléatoire de miel de fleurs. 
à larges feuilles présente en moyenne des dates de floraison décalées d'environ 12 jours entre 1950 et 1990.

Si le tilleul est le régal des abeilles, la production de miel de tilleul dans le Haut-Jura est une autre affaire. Des conditions climatiques favorables, des stations ensoleillées, des terrains plus ou moins humides favorisent une floraison optimale souvent accompagnée de miellée. Par ailleurs, il faut bien reconnaître qu'au-dessus de $1000 \mathrm{~m}$ les tilleuls ont été plantés (allées, bosquets, murgiers, arbres isolés près des habitations) et qu'ils sont par conséquent peu fréquents (fig. 5) comparés aux érables sycomores ou aux sorbiers (fig. 4).

Suivant les endroits et les saisons, les "petits trèfles blancs» (Trifolium montanum, T. repens, voire T. hybridum), par leur abondance et leur attractivité dans les prairies ou les pâturages, mais aussi dans les cultures avec tréfles en sous-semis (sans compter les cultures de tréfles proprement dites), dominent nettement les autres espèces à floraison tardive ou celles à deuxième floraison. A ce propos, BADOUX 1993, a montré que la proportion de tréfles blancs est nettement supérieure dans les cultures de fétuque des prés tétraploïde plutôt que diploïde.

Les tilleuls et trèfles appartiennent tous deux à ce qu'il est convenu d'appeler la végétation secondaire. Quant à la végétation primaire qui se limite ici au couvert forestier, elle ne comporte plus guère d'espèces mellifëres hormis le sapin blanc. La prenanthe, l'épiaire ou le séneçon (Prenanthes purpurea, Stachys silvatica, Senecio nemorensis) constituent un apport mellifëre négligeable. Par contre, la végétation tertiaire à proximité des habitations joue un rôle toujours plus grand (BĖGUIN 1993) au fur et à mesure de l'avancement de la période de végétation: mûrier, troène, spirées (Morus nigra, Ligustrum ovalifolium, Spiraea japonica, S. salicifolia, S. ulmifolia) sans oublier des especes très attractives comme la mauve, le roi des Alpes et surtout la bourrache (Malva alcea, Eryngium alpinum, Borago officinalis ${ }^{2}$ ).

Tout se passe comme si l'action - passée et présente - de l'homme sur la végétation naturelle potentielle était encore plus indispensable pour la production de miel en fin de saison dans le Haut-Jura.

\section{V) Mi-août/mi-octobre: récolte d'entretien}

Après la floraison des éricacées (Vaccinium myrtillus, V. uliginosum, V. vitis idaea, Calluna vulgaris), des framboisiers et des ronces (Rubus idaeus, $R$. caesius), la forêt ressemble vite à un désert mellifëre (les années sans miellat de sapin blanc).

Tous les produits nécessaires à l'entretien de la ruche se

V. Cinquième saison mellifère de MI-AOUT à MI-OCTOBRE : récolte pour l'entretien des ruches

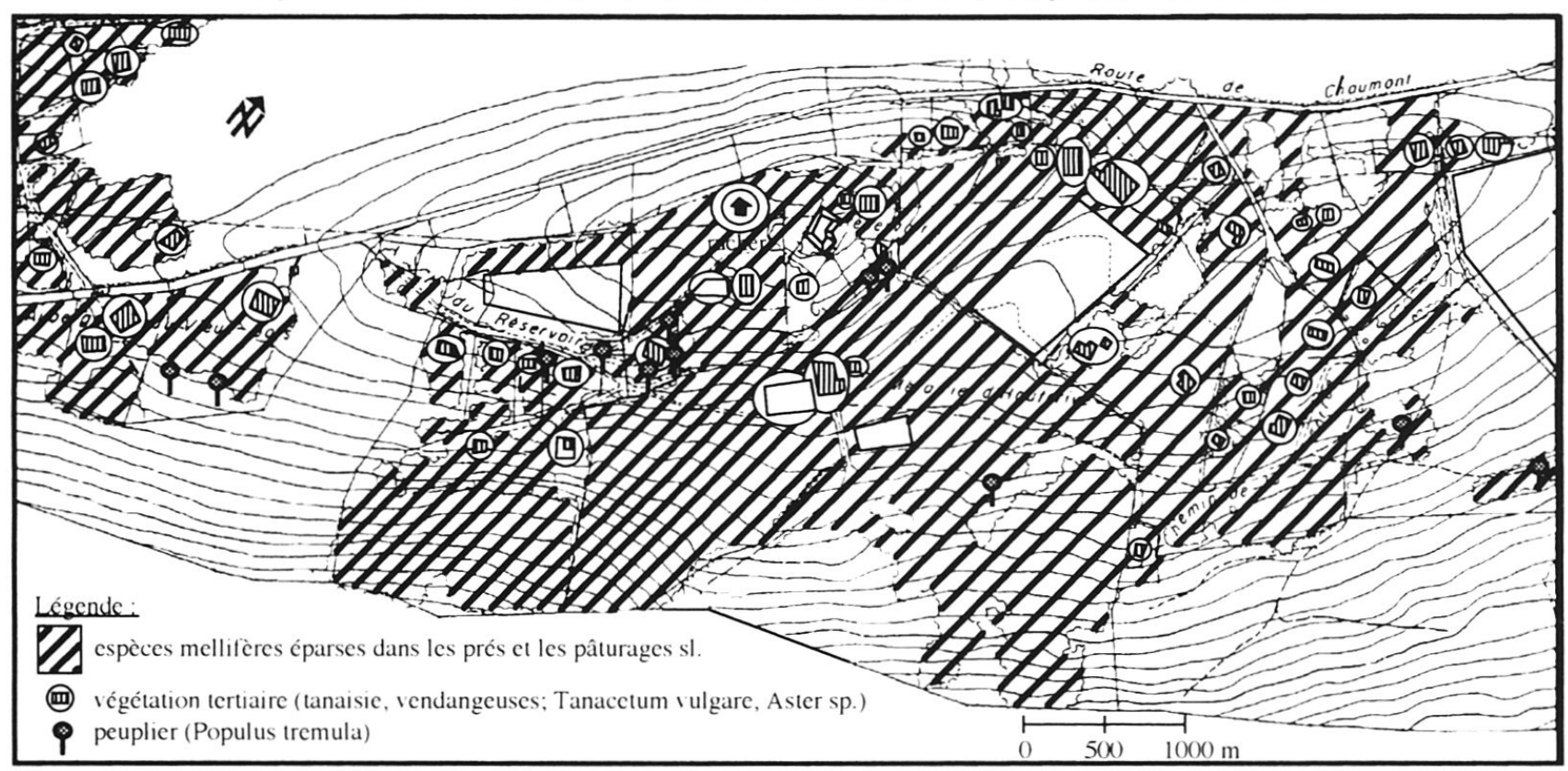

Fig. 6 Dès la mi-août et jusqu'à la fin de la période de végétation, la forêt et son manteau deviennent un véritable désert mellifère sauf en cas de miellat. Quant aux prairies et aux pâturages, ils apportent quelques colchiques d'automne, quelques astéracées, campanulacées et autres fabacées. Le pollen est surtout prélevé à proximité des habitations sur des espèces très attractives: bourrache, tanaisie, vendangeuse, rose trémière, cosmos, etc.

Comme source de propolis ne figure ici que le peuplier tremble; les ormes et les bouleaux sont relativement rares alors que les frênes, plus fréquents, sont apparemment de moindre intérêt. 
situent dès lors dans les pâturages (cirses, chardons, carline; Cirsium arvense, C. acaule, C. eriophorum, Carduus defloratus, Carlina acaulis) ou dans les prés (lotier, euphraise, colchique, trèfles; Lotus corniculatus, Euphrasia rostkoviana, Colchicum autumnale, Trifolium sp.) ou encore aux alentours des habitations (mûrier, bourrache, cosmos, tournesol, rose trémière, vendangeuses: Morus nigra, Borago officinalis, Cosmos bipinnatum, Helianthus annuus, Althaea rosea, Tanacetum vulgare, Aster amarellus) y compris les bords de chemin (solidage, épilobe, orpin; Solidago canadensis, Epilobium angustifolium, Sedum montanum).

En cette fin de saison, à quelques exceptions près, ces espèces sont généralement peu abondantes, dispersées et peu attractives.

Nous discuterons au chapitre 4 des possibilités et de l'opportunite de raccourcir cette dernière saison mellifëre en cultivant des plantes mellifëres à proximité des ruchers.

\section{VI) Juin/juillet/août/septembre: carte spéciale du miel de sapin}

Pour l'apiculteur des montagnes jurassiennes, le bon développement du sapin blanc est fondamental.

Suivant le mode d'exploitation forestier, suivant les fac- teurs climatiques et édaphiques du Haut-Jura, les strates arborescentes et arbustives présentent des degrés de recouvrement variables de sapins. Certains endroits du Risoux n'abritent que des forêts denses ou des pâturages boisés d'épicéas. Par contre, dans notre exemple de Chaumont, l'épicéa, le hêtre et le sapin sont parties à peu près égales. Mais chez ce dernier, la pyramide d'âge est essentiellement formée d'un mélange de jeunes et de vieux individus; les sapins dans la force de l'âge, entre 25 et 60 ans, sont moins bien représentés. D'après Pascal Junod, ingénieur forestier du canton de Neuchâtel (communications orales), le chevreuil et le bétail pourraient expliquer partiellement cette faible proportion de «perchis»: ces phénomènes de déséquilibre sylvo-cynétique qui apparaissent par endroits seraient dus à des dégâts d'abroutissement et secondairement de frayure et de piétinement.

Entre 1000 et $1200 \mathrm{~m}$, le sapin blanc se développe bien dans les lisières et les clairières, les anciens pâturages boisés, les murgiers et à proximité de certaines constructions y compris les voies de communication. Même si les forestiers ne favorisent pas actuellement le sapin blanc, ce dernier bénéficie néanmoins indirectement de l'influence humaine. A ce propos, on imagine souvent mal l'action passive des chiens qui, en éloignant de fortes populations de chevreuils friands de sapelots, limitent un sévère abroutissement. A titre indicatif, nous dénom-

VI. Saison mellifère spéciale du miellat de Buchneria pectinatae (JUIN) - JUULLET-AOUT- (SEPTEMBRE) production du miel de sapin

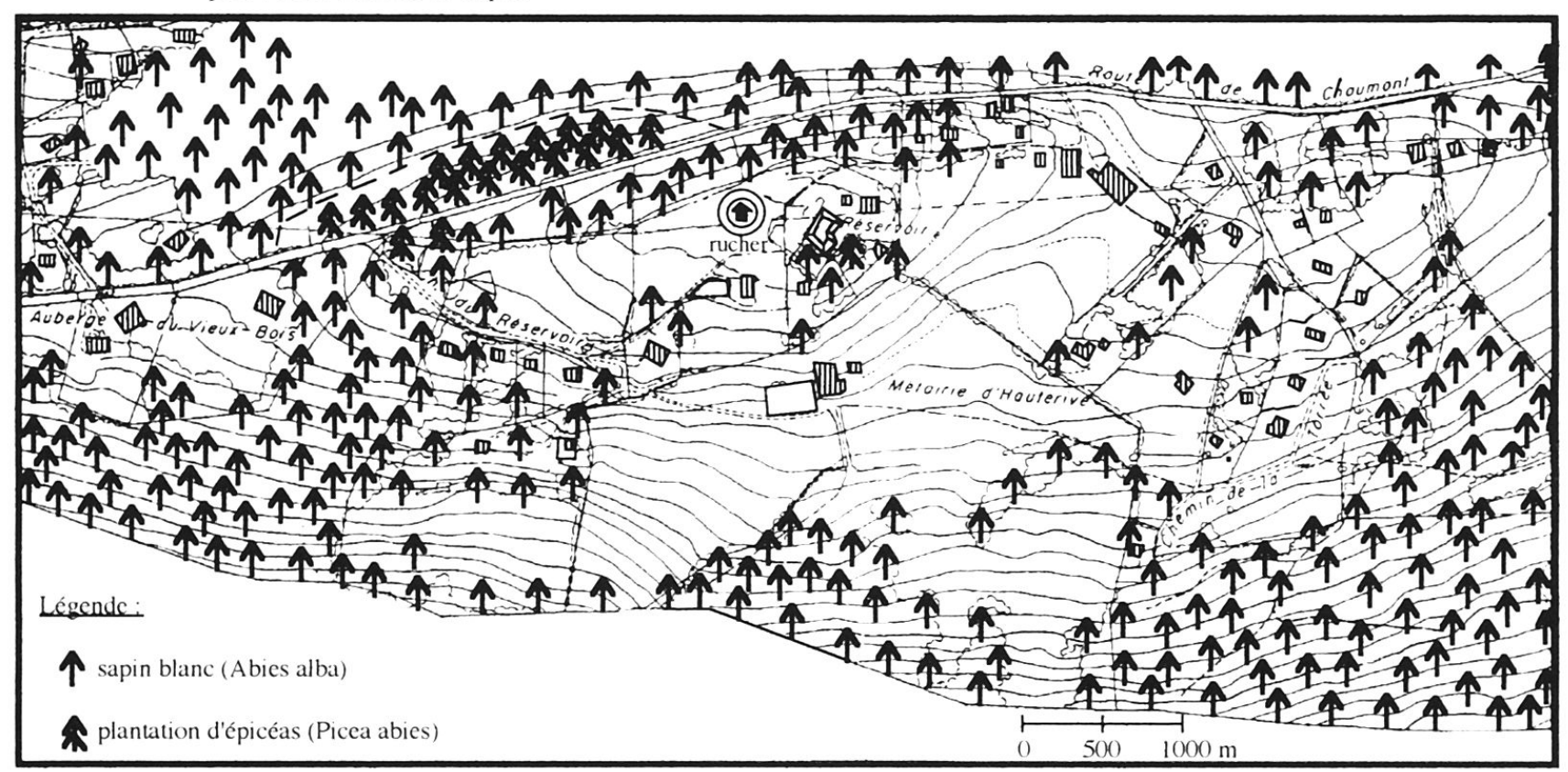

Fig. 7 Dans la région de Chaumont, le sapin blanc est omniprésent dans toute la zone forestière. Exception: certaines plantations d'épicéas. D'une manière générale, toute cette zone située au-dessus de $1000 \mathrm{~m}$ correspond à des pâturages plus ou moins abandonnés au cours du XXe siècle. Les lisières, les haies, les bosquets, les murgiers, les bords de chemin, la proximité des constructions sont des endroits de prédilection pour le sapin blanc. 
brons actuellement une douzaine de chiens sur notre diction.

En compétition avec le hêtre (Fagus silvatica) et l'épicéa (Picea excelsa), le sapin blanc (Abies alba) est devenu plus abondant, plus vigoureux et plus accessible pour les abeilles dans une végétation secondaire résultant d'une action anthropozoogène plus ou moins modérée.

\section{Discussion/conclusion}

Cinq saisons mellifëres peuvent être nettement différenciées dans le Haut-Jura; à l'exclusion de la période spéciale, aléatoire et intermittente (juin/juillet/août/septembre) du miel de sapin. En fait, une période un peu particulière, une sixième saison mellifère (début août/début septembre) pourrait être envisagée après la floraison du tilleul. Cette dernière saison correspondrait en quelque sorte à la floraison de champs de trèfles (Trifolium montanum, T. repens) et/ou de bourrache (Borago officinalis) et/ ou de phasélie (Phacelia tanacetifolia). Il s'agirait en d'autres termes de faire fleurir à une date avancée des espèces apiculturales attractives, groupées, et proches des ruches; seuls ces facteurs biologiques, écologiques et énergétiques permettraient une ultime récolte de miel de fleur avant le début de «l'indolence» des abeilles. Ces cultures tardives et sélectives sont d'autant plus à encourager qu'en août-septembre, les conditions climatiques peuvent être excellentes pour le travail des abeilles. Ces cultures présentent également l'avantage de combler une lacune en attendant un miellat tardif toujours possible; les chances de pullulation de Buchneria pectinatae diminuent normalement dès la mi-août comme l'a si bien démontré MAQUELIN (1978): les pucerons sont moins nombreux en moyenne (dernières générations, femelles ovipares adultes). Finalement, ces cultures d'automne revêtent d'autant plus d'importance que sans elles et sans miellat, la situation trophique peut devenir critique pour les ruches (BÉGUIN 1989).

Ces cinq ou six saisons melliferes se succèdent de façon quasi ininterrompues; certaines espèces de moindre intérêt apicole peuvent jouer un rôle de relais temporel en apportant un appoint non négligeable entre deux saisons melliferes fondamentales.

L'action de l'homme, en modifiant le paysage végétal originel n'induit donc pas de carences trophiques dans le Haut-Jura durant la période d'activité des abeilles. Au contraire, en transformant presque toute la végétation primaire en végétation secondaire et tertiaire, l'homme rend ce territoire plus mellifère. Tout se passe comme si dans un premier temps l'impact humain (déboisement et création de pâturages, de prairies, de groupements végétaux annexes) était indispensable pour toute production apicole. Tout se passe ensuite comme si une exploitation rationnelle, réfléchie et respectueuse de l'environnement devenait une condition nécessaire pour améliorer la production et la qualité du miel.
Inconsciemment, l'homme a construit un paysage mellifëre qu'il doit gérer maintenant consciemment.

Les ressources potentielles abondent sans discontinuer. Mais les quantités de produits prélevés durant les saisons sur les différentes espèces varient considérablement en fonction des conditions climatiques. Ce sont ces dernières qui déterminent la durée et l'intensité des floraisons et indirectement la préhension qualitative et quantitative des produits mellifères par les butineuses. C'est ainsi qu'au fil des saisons, le "mélange miel de fleurs» d'une part, le «mélange miel de fleurs-miel de sapin» d'autre part, varient considérablement. Les proportions de nectar prélevé sur les espèces végétales changent; un vent violent ou un gel tardif peuvent par exemple compromettre l'importante récolte de miel d'érable sycomore en cassant les fragiles jeunes grappes ou en gênant l'éclosion des fleurs. Une période de sécheresse ou une période pluvieuse limitent fortement la teneur en miel de tilleul ou de framboisier.

D'ailleurs dans un même rucher, le goût du miel n'est-il pas sensiblement différent d'une année à l'autre?

L'occupation de l'espace par les butineuses varie non seulement en fonction des 5 ou 6 saisons mellifères reconnues mais aussi en fonction de la répartition et de la valeur mellifere des especes, des associations et des complexes de végétation; quels que soient les différents niveaux d’intégration spatiale (flore, végétation, paysage), les ressources melliferes apparaissent sous trois formes principales: linéaire, étendue ou ponctuelle: linéaire par exemple en mars le long des haies et des lisières de noisetiers (fig. 2), étendue en juillet-août sur les surfaces forestières abritant du miellat de sapin blanc (fig. 7), ponctuelle en mars et en août autour des habitations (fig. 2 et 4 ) ou encore en avril avec les bosquets et les arbres isolés de saule marsault (fig. 3).

Selon les saisons, les abeilles se cantonnent donc dans des zones mellifëres différentes. Elles ont même des endroits de prédilection sur une même espèce selon l'état physiologique et phénologique de la plante. Au cours d'une même journée, sur les énormes tilleuls de la métairie d'Hauterive par exemple, nous avons observé des concentrations variables en fonction de l'exposition et de la hauteur des arbres.

Si l'on considère que la surface cartographiée est plus ou moins représentative des régions habitées du Haut-Jura central (entre 1000 et 1300 m d'altitude), on peut estimer assez rapidement l'environnement apicole d'un rucher.

Cependant, un examen plus approfondi devrait être envisagé dans les régions riches en éricacées (Vaccinium, CalIuna), en dipsacacées (Succisa, Knautia, Scabiosa) ou en rhamnacées (Frangula, Rhamnus); plus précisément dans les zones tampons entre les prés et les tourbières où l'on observe un nombre élevé d'espèces en fleur tard dans la période de végétation (juillet/août). Il s'agit là de paysages végétaux mellifëres spécialisés (hypogéosigmassociations de THEURILLAT 1992a, 1992b, de BÉGUIN \& al. 1993). Ces auteurs ont montré également les relations étroites existant entre certaines populations animales et 
certaines unités du paysage végétal (géosyntaxons). En ce qui concerne les abeilles, nous constatons que:

1. L'unité de paysage végétal correspondant le mieux à l'aire de butinage des abeilles d'un rucher du Haut-Jura est la géosigmassociation.

2. Les zones de contact entre différentes géosigmassociations constituent des endroits de prédilection pour l'emplacement des ruchers. Les zones de contact entre unités inférieures de paysage (macrosigmassociation et hypogéosigmassociation) sont également des zones mellifères privilégiées.

3. Le complexe de végétation mellifère idéal dans le Haut-Jura est composé d'une mosaïque de sigmassociations primaires (forêts mixtes, environ 45\%) de sigmassociations secondaires (prairies, pâturages, environ $45 \%$ ) et de sigmassociations tertiaires (abords d'habitations et cultures mellifëres, 1-10\%).

La valeur mellifère de n'importe quelle contrée peut donc être obtenue en dressant les cartes des saisons mellifères selon le modèle des figures 2 à 7 . On peut y déceler d'éventuelles carences quantitatives et/ou qualitatives à tel ou tel endroit et à tel ou tel moment. Lorsque l'apiculteur a pris conscience de l'existence d'un «désert biologique» ou d'une période creuse, il palie à ces lacunes par des moyens appropriés: déplacement des ruches, apport judicieux de pollen, dégagement des meilleurs arbres mellifëres (tilleuls, érables, sapins, saules marsault, merisiers, alisiers blancs, sorbiers des oiseleurs), plantations d'arbustes jouant le rôle de relais ou d'appoint (sureau noir, spirée, fruitiers divers), remplacement partiel des cultures subventionnées d'orge et d'avoine par des cultures de trèfles blancs, et/ou de phasélie et/ou de bourrache (tous les trois ans), entretien des haies et des lisières diffuses où sont favorisées des espèces mellifères telles que le noisetier, l'aubépine, le framboisier, la bourdaine, etc.

L'établissement des cartes de saisons mellifères est simple puisqu'il ne fait intervenir qu'une quinzaine d'espèces végétales facilement identifiables n'importe quand. Il est donc souhaitable que de telles cartes soient établies à proximité des ruchers ou près des emplacements prévus pour l'installation de colonies.

La méthode proposée aide à résoudre des problèmes de transhumance. Elle autorise une estimation de la charge possible en ruches. Elle favorise une meilleure conduite du rucher (BERTRAND 1989). Nous espérons globalement qu'elle permette d'envisager une exploitation et une gestion rationnelles du territoire.

Le réchauffement du climat prévu pour ces prochaines décennies pourrait favoriser l'apiculture dans le HautJura en facilitant le déplacement des abeilles et en augmentant la vitalité de la majorité des meilleures espèces mellifères.

\section{Essai de vocabulaire}

Association végétale mellifère: association abritant une ou plusieurs espèces mellifères. Exemple: l'Abieti-Fagetum est une association végétale mellifère de juin à septembre. Le Fagetum typicum est une association végétale peu mellifère. Un champ d'orge sans trèfles blancs en sous-semis n'est pas une association végétale mellifère. Complexe de végétation mellifère: complexe abritant une ou plusieurs associations végétales mellifères. Exemple: l'Abieti-Fageto-sigmetum dès la fin de l'hiver jusqu'à fin septembre est une sigmassociation mellifère. Il s'agit d'un paysage forestier composé d'un complexe de végétation comprenant non seulement une association climacique primaire (Abieti-Fagetum) mais aussi des groupements secondaires, voire tertiaires (groupements de manteau, d'ourlet, de bord de chemin ou de tourne-char, de clairières et de différents stades de recolonisation après les tempêtes ou les coupes rases). Dans les complexes de végétation mellifères, on peut distinguer plusieurs types tels que la sigmassociation mellifère, la géosigmassociation mellifère, etc. Un complexe de végétation s. l. est l'ensemble des groupements végétaux sur une partie de territoire d'extension spatiale variable.

\section{Notes}

1 A notre connaissance, la toxicité des tilleuls vis-à-vis des abeilles reste encore énigmatique. Récemment, Philippe KÜPFER, directeur de I'Institut de botanique de Neuchâtel, nous signalait (communication orale) la présence de quelques dizaines de bourdons morts sous un tilleul argenté au jardin botanique (juillet 1993).

2 Selon COUPLAN (1983), la bourrache officinale, originaire de l'Europe méridionale, est cultivée depuis fort longtemps comme plante médicinale, alimentaire, ornementale et mellifère. Elle est subspontanée sur une grande partie de notre continent. Les jeunes feuilles, le sommet des tiges et les fleurs d'un magnifique bleu d'azur sont comestibles crues: elles ont un goût rafraîchissant qui rappelle le concombre. Plus vieilles, les feuilles seront meilleures cuites, car elles sont alors couvertes de poils rigides qui peuvent irriter la peau (cela est également vrai des tiges) et leur texture est assez désagréable quand elles sont crues. On en fait encore parfois des beignets dans le pays niçois. En Grèce, elles servaient à envelopper des boulettes farcies. On a conservé au vinaigre les feuilles de la bourrache en Europe et en Inde. Les anciens Grecs utilisaient déjà les fleurs pour parfumer leurs boissons. L'usage s'en est perpétué jusqu'à nos jours. La plante contient des mucilages, du tanin, de la saponine, du nitre, du potassium et un peu d'huile essentielle. Tiges et feuilles sont diaphorétiques et diurétiques. Les fleurs sont émolientes, expectorantes et légèrement laxatives. 


\section{Bibliographie}

AESCHIMANN, D. \& BURDET, H. (1989): Flore de la Suisse; le nouveau Binz. Griffon, Neuchâtel, 597 pp.

BADOUX, S. (1993): Pourquoi pas une fétuque des prés tétraploïde? Dans: Revue suisse Agric., Vol. 25, no 4, 211-213.

BALAYER, M. (1990): Evaluation des potentialités mellifères en Roussillon. Dans: Bull. Soc. bot. Fr., 137, Lettres bot. (2/3), 157-171.

BÉGUIN, C. (1989): Faible productivité de miel dans le Jura en 1988: facteurs trophiques ou climatiques? Dans: Journal suisse d'apiculture, no 3, 73-79.

- (1990): Evolution de la flore mellifère du Haut-Jura. Dans: Revue horticole suisse, Vol. 63, no 7/8, 175-180.

- (1993): Les espèces mellifères introduites dans le HautJura présentent-elles un apport intéressant? Dans: Revue suisse, Agric. Vol. 25, no 4, 251-245.

BÉGUIN, C., GRANDTNER, M. \& GERVAIS, C. (1994): Analyse symphytosociologique de la végétation littorale du SaintLaurent près de Cap Rouge, Québec. Dans: Phytocoenologia 24, 27-52.

BERTRAND, E. (1983): La conduite du rucher. Payot, Lausanne, $304 \mathrm{pp}$.
BRIANE, G. \& CABROL, J.-L. (1986): L'abeille dans le géosystème: essai de cartographie des ressources mellifères. Dans: Rev. géogr. des Pyrénées et du Sud-Ouest, tome 57, fasc. 3, 363-373.

COUPLAN, F. (1983): Le régal végétal. Dans: Encyclopédie des plantes comestibles de l'Europe. Vol. 1. Debard, Paris, $453 \mathrm{pp}$.

DEFILA, C. (1992): Phänologie, ein Indikator für Umweltveränderungen. In: Schweiz. Rundschau Med. (PRAXIS) 81 Nr. 11, 343-346.

GEHU, J.-M. (1979): Essai d'évaluation phytocœnotique de l'artificialisation des paysages. Dans: Séminaire de phytosociologie appliquée, Indices biocœnotiques, Metz, 95-118.

MAQUELIN, CH. (1974): Observations sur la biologie et l'écologie d'un puceron utile à l'apiculture: Buchneria pectinatae. Dans: Thèse EPF Zurich, 120 pp.

PRIMAULT, B. (1971): Atlas phénologique. Institut suisse de météorologie. Zurich. Troisième édition.

THEURILLAT, J.-P. (1992a): Etude et cartographie du paysage végétal (Symphytocœnologie) dans la région d'Aletsch (Valais, Suisse). Dans: Beitr. geobot. Landesaufn. Schweiz 68, $384 \mathrm{pp}$. + cartes et tableaux

- (1992 b): L'analyse du paysage végétal en symphytocœnologie: ses niveaux et leurs domaines spatiaux. Dans: Bull. Ecol. 22, 103-147. 